\title{
A simple serum depletion method for proteomics analysis
}

\author{
Alexandre Zougman*, 1 iD, John P Wilson ${ }^{2}$ \& Rosamonde E Banks ${ }^{1}$ \\ ${ }^{1}$ Clinical \& Biomedical Proteomics Group, Leeds Institute of Medical Research, St James's University Hospital, Leeds, LS9 7TF, UK; ${ }^{2}$ Protifi LLC, PO Box 2317, Huntington, \\ NY 11743, USA; *Author for correspondence: a.zougman@leeds.ac.uk
}

BioTechniques 69: 149-151 (August 2020) 10.2144/btn-2020-0017

First draft submitted: 17 February 2020; Accepted for publication: 6 April 2020; Published online: 6 May 2020

\section{ABSTRACT}

Serum is the body fluid most often used in biomarker discovery. Albumin, the most abundant serum protein, contributes approximately $50 \%$ of the serum protein content, with an additional dozen abundant proteins dominating the rest of the serum proteome. To profile this challenging protein mixture by proteomics, the abundant proteins must be depleted to allow for detection of the low-abundant proteins, the primary biomarker targets. Current serum depletion approaches for proteomics are costly and relatively complex to couple with protein digestion. We demonstrate a simple, affordable serum depletion methodology that, within a few minutes of processing, results in two captured serum fractions - albumin-depleted and albumin-rich - which are digested in situ. We believe our method is a useful addition to the biomarker sample preparation toolbox.

\section{METHOD SUMMARY}

We describe a method for simple albumin depletion by serum fractionation coupled with proteomics analysis. The method is based on selective protein adsorption to quartz under basic conditions. While many serum proteins are captured and can subsequently be eluted, albumin and some other proteins are not retained and pass through the quartz trap. The flow-through albumin-rich fraction is diluted with methanolic ammonium acetate solution and captured in a second quartz trap. Both fractions are digested in situ for proteomics analysis.

\section{KEYWORDS:}

albumin depletion • serum protein fractionation • serum proteomics • SiTrap • STrap

In translational medical research, serum is a valuable source of protein biomarker molecules. However, common abundant serum proteins interfere with identification of the low-level proteins of interest by proteomics and need to be depleted from the serum samples to increase the protein identification efficiency. Albumin is the most abundant serum protein, constituting approximately $50 \%$ of the total serum proteome [1]. Current serum depletion methods, such as those based on affinity removal, are expensive and relatively complex in the downstream hyphenation with proteomics processing [2]. Recently we described two bottom-up proteomics sample preparation methods, STrap (Suspension Trapping) and SiTrap (Simultaneous Trapping), based on protein capture and digestion in situ in quartz and cellulose depth filter traps [3,4]. While working with serum samples, we noticed that at basic pH (e.g., diluted in 20 mM triethylammonium bicarbonate [TEAB] buffer), serum albumin is not trapped by quartz depth filters but many other serum proteins are captured (Figure $1 \mathrm{~A}$ ). One of the possible explanations for the behavior of albumin is that its isoelectric point is 4.7. At basic $\mathrm{pH}$, albumin is negatively charged; as the silica surface is also negatively charged at this $\mathrm{pH}$, albumin would not be retained by the quartz filter due to electrostatic repulsion. The simple serum fractionation results in two protein fractions. The captured fraction, depleted of albumin, is directly digested in the trapping unit, while the flow-through 'albumin-rich' fraction is diluted with six volumes of $30 \mathrm{mM}$ ammonium acetate in methanol and subsequently captured and digested in another trapping unit (Figure 1B).

To evaluate this approach further, $0.5 \mu$ of normal human serum was either digested directly by the STrap technology (six replicate samples in total) or diluted with $20 \mathrm{mM}$ TEAB buffer and processed by fractionation as described above using trapping quartz tips, resulting in two fractions, the captured and the flow-through (three replicates for each fraction, six samples in total). The results of mass spectrometry analysis using each approach were then merged. The fractionation approach led to an increase of approximately $32 \%$ in protein identifications compared with the direct approach (242 vs 183 proteins, respectively) (Figure 2A \& B). In addition, combined digests of the three 'depleted' fractions were compared with the combined three 'direct' digests. An increase in protein identifications of $28 \%$ was observed for the 'depleted' fractions (218 vs 170 proteins, respectively), proving that most identifications come from the 'depleted' fraction. It is worth noting that the simple strategy used for capture and digestion of the flow-through serum albumin fraction - sample dilution in six volumes of methanolic ammonium acetate, protein trapping, reduction/alkylation and digest - could also be utilized for processing of less complex protein mixtures such as antibody preparations. Even though simple in execution, the proposed method is a useful, practical and rapid sample preparation tool for a proteomic biomarker laboratory. 


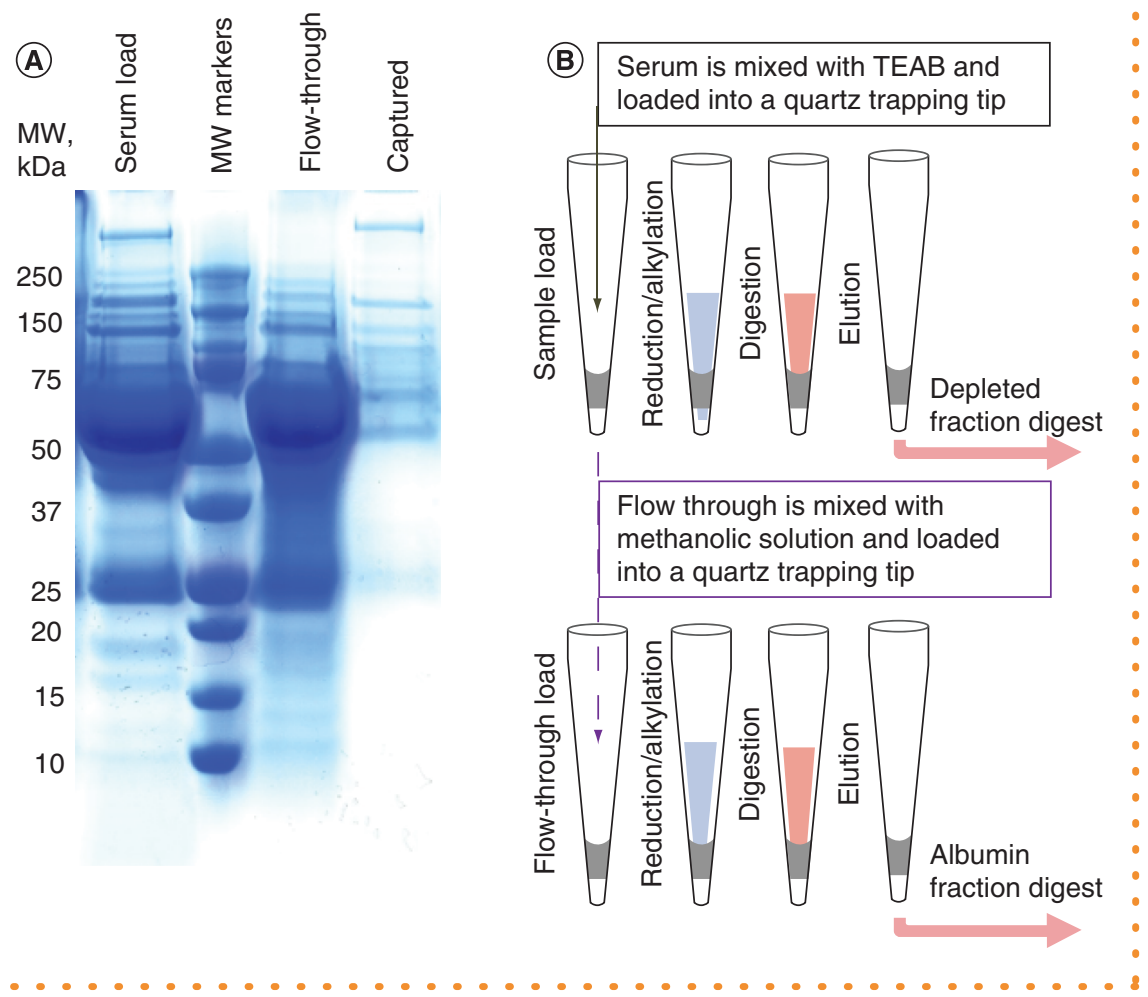

Figure 1. In-tip serum fractionation based on selective protein adsorption on quartz. (A) The simple serum fractionation approach using quartz tips produces two fractions, one of which is devoid of albumin. The loaded serum, captured and flow-through fractions were run on NuPAGE 4-12\% Bis-Tris protein gel and stained with InstantBlue Coomassie stain. (B) Schematics of the proposed serum fractionation and digestion methodology. A serum sample is diluted with TEAB buffer and loaded into a quartz trapping tip. The bound proteins are reduced and alkylated in situ. After washing, digestion is performed by introducing a trypsin solution into the tip. The uncaptured flow-through fraction, in turn, is diluted with methanolic solution of ammonium acetate and loaded into another quartz tip, where the proteins are also trapped, reduced/alkylated, and digested in situ. MW: Molecular weight; TEAB: Triethylammonium bicarbonate.

\section{Trapping units}

Trapping tips were constructed by inserting quartz depth filter plugs into $200-\mu l$ pipette tips as described previously $[3,5]$. For the sample processing steps involving centrifugation (load, wash and elution), the tips were placed in 2.0- or 1.5-ml sample tubes with the aid of tube adapters [3].

\section{Serum processing}

A sample of normal human serum was obtained from the Leeds Multidisciplinary Research Tissue Bank. $0.5 \mu l$ of the serum was either processed directly by the OQ STrap (Only Quartz Suspension Trapping) protocol [5] in the trapping tips using serum solubilization with $25 \mu \mathrm{l}$ 5\% SDS in $50 \mathrm{mM}$ Tris- $\mathrm{HCl}, \mathrm{pH} 7.6$ and in situ tryptic digestion (six replicate samples in total), or was processed utilizing the proposed method, in other words, diluted with $30 \mu \mathrm{l}$ of $20 \mathrm{mM}$ TEAB buffer and processed by fractionation in the trapping tips, resulting in two fractions, 'captured' (albumin-depleted) and 'flow-through' (albumin-rich) (three replicates for each fraction, six samples in total). For the proposed methodology, the serum diluted in TEAB buffer was loaded into the trapping tip and gently pushed through with the aid of a syringe with a tip adapter; the 'flow-through' fraction was collected. The tip with the 'captured' fraction was then inserted into a $2.0-\mathrm{ml}$ sample tube and washed consecutively with 100 and $40 \mu \mathrm{l}$ of $20 \mathrm{mM}$ TEAB using centrifugation at $2500 \times \mathrm{g}$. Reduction/alkylation and digestion of the trapped proteins were performed in situ as previously described for the SiTrap processing [4]. The 'flow-through' fraction was diluted with six volumes of $30 \mathrm{mM}$ ammonium acetate in methanol (prepared by mixing $1 \mathrm{M}$ ammonium acetate solution with anhydrous methanol) before being trapped and digested in another trapping tip in the same way as the 'captured' fraction.

\section{Proteomics analysis}

The resulting peptides were separated online by reversed-phase capillary liquid chromatography using an EASY-nLC 1000 system (Proxeon) connected to a custom-made 30-cm capillary emitter column (inner diameter $75 \mu \mathrm{m}$, packed with $3-\mu \mathrm{m}$ Reprosil-Pur $120 \mathrm{C}_{18}$ media, Dr Maisch). The EASY-nLC 1000 system was coupled with a linear ion trap- orbitrap (LTQ-Orbitrap ${ }^{T M}$ ) Velos mass spectrometer (Thermo). The acquisition time was $90 \mathrm{~min}$ and the main part of the gradient was $3-22 \%$ acetonitrile in $0.1 \%$ formic acid. Survey mass spectrom- 


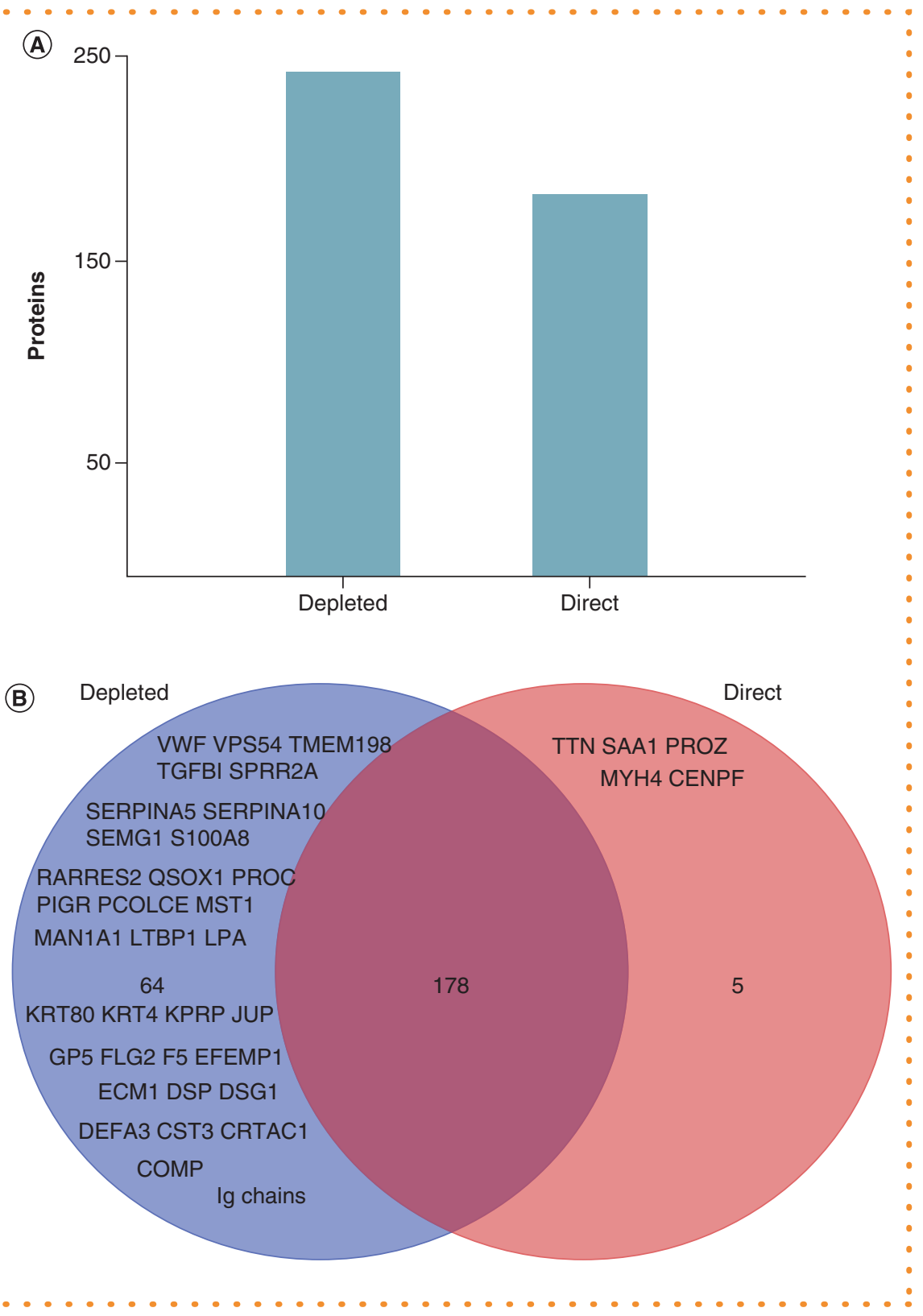

Figure 2. Serum protein identification by the fractionation and direct approaches. The fractionation approach leads to an increase of $\sim 32 \%$ in protein identifications. (A) Histogram showing comparison of fractionation serum processing with the direct serum digest method. Totals represent proteins identified with at least two peptides. (B) Venn diagram showing proteins identified using the fractionation serum processing versus the direct serum digest method (proteins identified with at least two peptides).

etry scans (scan range of 305-1350 amu) were acquired in the Orbitrap with a resolution of 60,000. The most intense ions (up to 20 per scan) were fragmented and analyzed in the ion trap. Data were processed against a Uniprot human database (October 2018) with the MaxQuant 1.5.2.8 software package [6,7]. Carbamidomethylation of cysteine was set as a fixed modification, with protein $\mathrm{N}$-terminal acetylation and oxidation of methionine as variable modifications. The maximum protein and peptide false discovery rates were set to 0.01. Only proteins identified with at least two peptides were considered.

\section{Future perspective}

In this work we present a simple, streamlined concept for fractionation-capture and in situ digestion of serum proteins for bottom-up proteomics analysis. We believe our methodology could be of practical utility to scientists engaged in clinical proteomics research and serum or plasma biomarker profiling. To remove the bottleneck of having to make the protein trapping units in-house to ascertain their 
reproducible performance, the units, in different formats and capacities, will be made commercially available. Such availability is key for the wide acceptance of the method.

\section{Author contributions}

A Zougman developed the methodology; J Wilson contributed to the method's development; A Zougman designed and performed the experiments; A Zougman and R Banks analyzed the data; A Zougman, J Wilson and R Banks wrote the manuscript.

\section{Financial \& competing interests disclosure}

A patent application related to this work was filed by Protifi, LLC. The authors have no other relevant affiliations or financial involvement with any organization or entity with a financial interest in or financial conflict with the subject matter or materials discussed in the manuscript apart from those disclosed.

No writing assistance was utilized in the production of this manuscript.

\section{Open access}

This work is licensed under the Attribution-NonCommercial-NoDerivatives 4.0 Unported License. To view a copy of this license, visit http://creativecommons.org/licenses/by-nc-nd/4.0/

\section{References}

1 Anderson NL, Polanski M, Pieper R et al. The human plasma proteome: a nonredundant list developed by combination of four separate sources. Mol. Cell Proteomics 3(4), 311-326 (2004).

2 Bjorhall K, Miliotis T, Davidsson P. Comparison of different depletion strategies for improved resolution in proteomic analysis of human serum samples. Proteomics 5(1), 307-317 (2005).

3 Zougman A, Selby PJ, Banks RE. Suspension trapping (STrap) sample preparation method for bottom-up proteomics analysis. Proteomics 14(9), 1006-1000 (2014).

4 Zougman A, Wilson JP, Roberts LD, Banks RE. Detergent-free simultaneous sample preparation method for proteomics and metabolomics. J. Proteome Res. doi:10.1021/acs.jproteome.9b00662 (2019) (Epub ahead of print).

5 Zougman A, Banks RE. C-STrap sample preparation method - in-situ cysteinyl peptide capture for bottom-up proteomics analysis in the STrap format. PLoS ONE 10(9), (2015).

6 Cox J, Mann M. MaxQuant enables high peptide identification rates, individualized p.p.b.-range mass accuracies and proteome-wide protein quantification. Nature Biotechnol. 26(12), 1367-1372 (2008).

7 MaxQuant 1.5.2.8 software package. http://www.maxquant.org 